\title{
Dynamical formation of stable irregular transients in discontinuous map systems
}

\author{
Hailin Zou, ${ }^{1}$ Shuguang Guan, ${ }^{2,3,4}$ and C.-H. Lai ${ }^{1,4}$ \\ ${ }^{1}$ Department of Physics and Centre for Computational Science and Engineering, \\ National University of Singapore, Singapore 117543 \\ ${ }^{2}$ Institute of Theoretical Physics and Department of Physics, \\ East China Normal University, Shanghai, 200062, P. R. China \\ ${ }^{3}$ Temasek Laboratories, National University of Singapore, Singapore 117508 \\ ${ }^{4}$ Beijing-Hong Kong-Singapore Joint Center of Nonlinear and Complex systems (Singapore), Singapore 117508
}

(Dated: November 20, 2018)

\begin{abstract}
Stable chaos refers to the long irregular transients, with a negative largest Lyapunov exponent, which is usually observed in certain high-dimensional dynamical systems. The mechanism underlying this phenomenon has not been well studied so far. In this paper, we investigate the dynamical formation of stable irregular transients in coupled discontinuous map systems. Interestingly, it is found that the transient dynamics has a hidden pattern in the phase space: it repeatedly approaches a basin boundary and then jumps from the bundary to a remote region in the phase space. This pattern can be clearly visualized by measuring the distance sequences between the trajectory and the basin boundary. The dynamical formation of stable chaos originates from the intersection points of the discontinuous boundaries and their images. We carry out numerical experiments to verify this mechanism.
\end{abstract}

PACS numbers: 05.45.-a,87.19.1j

\section{INTRODUCTION}

Usually, nonlinear dynamical systems may have stable attractors such as fixed points, limited cycles, or chaotic attractors. Apart from these, interestingly, it is shown that irregular long transients can also occur in such systems. For continuous dynamical systems, these irregular long transients are usually due to the existence of highdimensional chaotic saddles in phase space (see recent review [1] for details). These chaotic saddles often appear after crisis bifurcation [2]. The system may spend extremely long time in the vicinity of the chaotic saddle, and behaves as irregular as chaotic. Due to this reason, this type of irregular transients is usually called transient chaos, which is sensitive to the initial condition and thus have a positive largest Lyapunov exponent. For example, in Ref. [3], it is found that the development of transient chaos is related to the unstable-unstable pair bifurcation which involves an unstable periodic orbit in the chaotic attractor and another one on the basin boundary. Moreover, another interesting finding along this line is the super-transient whose average lifetime could be very long even far from the bifurcation point [4, [5]. Such supertransients have also been found in stochastic dynamical systems [6, 7].

However, there exists another distinct type of irregular transient that has negative largest Lyapunov exponent, usually occurring in discontinuous systems. This phenomenon was first observed in the coupled map lattice [8]. The complex transients behave irregularly with exponential decay of correlation both in time and space [9]. In addition, the transient time usually grows exponentially with system size which makes the attractors unreachable in large systems. Due to these properties, the transient is termed as stable chaos [9, 10]. Later, stable chaos was also reported in various types of dynamical systems [11 13]. Recently, it was found in the pulsecoupled oscillators systems which are frequently used to model neuronal activity [14 16]. In all the above works, the stable chaos appears in discontinuous map systems (or discontinuous return maps). Interestingly, it is found that the stable chaos could also appear in the continuous map system where there exists a transition from the standard chaos to the stable chaos [17].

There are some efforts attempting to illustrate the mechanism underlying the formation of stable chaos. For example, in Ref. [8], it was conjectured that the stable chaos is due to the hierarchical organization of subbasins in phase space. The subbasins are subspaces of a basin separated by walls through which an orbit cannot pass except at portals. The irregular transient is regarded as a sequence of transitions through a hierarchy of subbasins. However, the formation of these subbasins and portals is still not well understood. In Ref. [18], the stable chaos was attributed to the ordinary chaos in a continuous system slightly altered from the original discontinuous system. One deficiency of this approach is that chaos can exist even in a one-dimensional continuous map, while stable chaos typically happens in highdimensional dynamical systems. In addition, Ref. 10] showed that the alteration could be too large for some systems. It was shown that the stable chaos is analogous to deterministic cellular automata [9]. Along this line, the stable chaos was attributed to the nonlinear propagation of finite disturbances from the outer regions [10], and a stochastic model was presented to understand the mechanism of this nonlinear information flow [12]. However, the direct basic mechanism is still unclear. In spite of the works mentioned above, the dynamical formation of stable chaos has not been well studied to date and the 
mechanism is still unclear. In particular, how the stable chaos develops from the governing dynamical equations is not fully understood. One possible reason for this is that these global behaviors usually occur in high-dimensional dynamical systems, which is usually difficult to deal with mathematically.

In this paper, we focus on the problem on the dynamical formation of stable chaos in the discontinuous systems. For these systems, it is natural to relate the occurrence of stable chaos to the discontinuity of the local dynamics of the coupled dynamical systems. Motivated by this idea, in this paper, we directly investigate some coupled discontinuous map systems, which are different from the previous works. Our particular interest is to reveal how the discontinuity in the local dynamics of a coupled system can induce stable chaos. To this end, we specifically construct dynamical models whose local maps have only contracting pieces with absolute determinant smaller than 1 . As a consequence, the occurrence of chaos is excluded in such systems, and the generated long irregular transient is stable chaos by nature.

The organization of this papers is as follows. In Sec. II, we show that accompanying stable chaos, a regular pattern always exists in both coupled discontinuous maps and pulse coupled oscillators. In Sec. III, the dynamical origin of stable chaos is analyzed and verified by a twodimensional map. Finally, conclusions are drawn in the last section.

\section{THE REGULAR PATTERN ACCOMPANYING STABLE CHAOS}

In continuous dynamical systems, the stable manifolds of the saddle periodic orbits compose the basin boundaries. However, for discontinuous map systems with only contracting local dynamics, the basin boundaries could only include the set of points whose dynamics are discontinuous. This set of points comprises the pre-images of the discontinuous boundaries and the discontinuous boundaries themselves. Intuitively, the stable chaos might be related to this set. Normally, we cannot obtain this set directly because of the high dimensionality of the phase space. However, the basin boundaries can be easily detected. We can measure the distance of each point in the trajectory to the basin boundaries. Therefore, for each transient trajectory, we will have a corresponding distance sequence.

The distance of a point $x$ to the basin boundaries $B$ is defined to be $d=\min \|x-y\|_{2}$, where $y \in B$. This distance $d(x)$ also quantifies the degree of stability of the system under finite perturbation at a given point $x$. If the perturbation added to the system is larger than $d(x)$, the system will jump to another attractor. In this sense, the distance is the maximal finite perturbation that the system can tolerate without losing the stability. We use a simple procedure to obtain this distance. The steps are: (1) Randomly sprinkle many initial points, for example
200 , in a hypersphere with center $x$ and radius $r$. Initially $r$ is set to be a large value. If all the initial points settle onto the same attractor as the center $x$, go to step (3). (2) Set the new radius $r$ to be the minimum distance between the center and the set of initial points who have different final attractor from the center. (3) If $r$ does not change in the sequential $m$, say 5 , times, stop. Otherwise repeat the above steps.

To illustrate this idea, we first consider the following coupled map lattice with a periodic boundary [8]:

$$
x_{i}(n+1)=\frac{1}{2 r+1} \sum_{j=-r}^{r} f\left(x_{i+j}(n)\right),
$$

where the local dynamics is $f(x) \cong s x+\omega(\bmod 1)$. When $s$ is smaller than 1, the system (1) will finally approach a periodic attractor because of the negative Lyapunov spectrum. We choose $s=0.9$, and $\omega=0.118$ in this paper. We consider nearest-neighbor coupling: $r=1$. The number $N$ of oscillators is 28 , for which the irregular long transient is prominent. For the individual local map, there is a discontinuous boundary at $x=(1-\omega) / s=$ 0.98 .

Fig. 1(a) shows a typical stable irregular transient in this system. Its corresponding distance sequence to the basin boundaries is shown in Figs. 1(b) and 1(c). To our surprise, we find a regular pattern in the distance sequence despite of the irregular transient dynamics. As clearly shown in Fig. 1(c), the distance sequence first gradually approaches the basin boundaries. Then it jumps to some remote regions in the phase space and begin to approach the basin boundaries once again. During the whole transient period, such pattern repeatedly occurs until finally the system settles down on the trivial attractors.

Similarly, the regular pattern in the distance sequence can also be observed in the inhibitory pulse-coupled oscillators. This model describes $N$ oscillators, such as neurons, interacting on a direct network by sending and receiving pulses [15, 19, 22, 23]. The state of each oscillator $i$ is specified by a phase-like variable $\phi_{i}(t) \in(-\infty, 1]$. The dynamics of the single oscillator $i$ is given by

$$
d \phi_{i} / d t=1
$$

when $\phi_{i}(t)$ reaches a phase threshold 1 , this phase is reset to zero, $\phi_{i}\left(t^{+}\right)=0$, and a pulse is generated. After a delay time $\tau$ this pulse is received by all oscillator $j$ having an in-link from $i$. This induces a phase jump in $j$ according to

$$
\phi_{j}(t+\tau)^{+}=\min \left\{U^{-1}\left(U\left(\phi_{j}(t+\tau)\right)+\epsilon_{j i}\right), 1\right\},
$$

where the function $U$, which determines the phase jump, is twice continuously differentiable, monotonically increasing, concave and normalized $(U(0)=0$ and $U(1)=$ 1). Furthermore, the coupling strength is also normalized as $\epsilon_{i j}=\epsilon / k_{j}$,where $k_{j}$ represents the number of incoming links of node $j$ (in-degree in graph term). This model is 


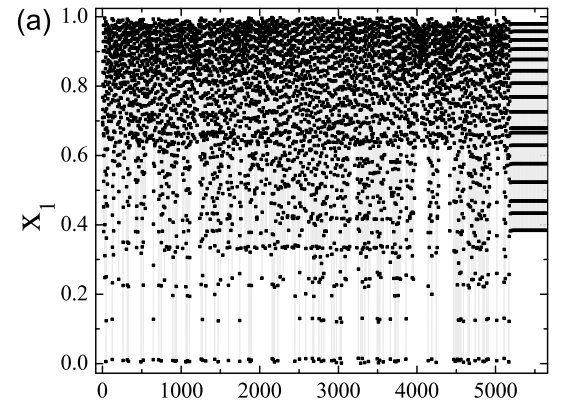

n
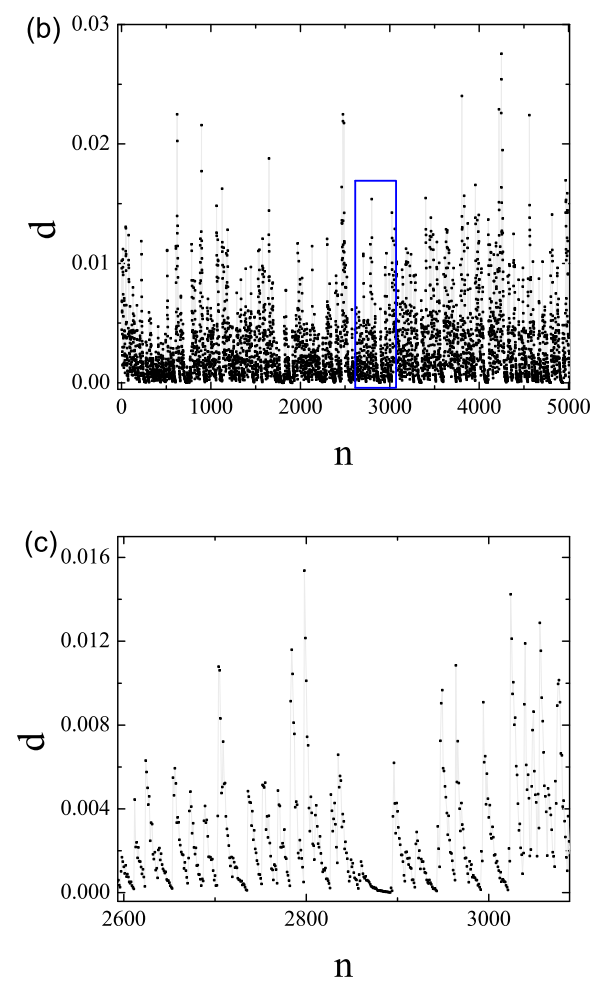

FIG. 1: (a) The long irregular transients observed in Eq. (1) for $x_{1} . \quad n$ is the time step. (b) The corresponding distance sequence $d$ to the basin boundaries. (c) The enlargement of the rectangle in (b).

equivalent to the standard leaky integrate-and-fire model with $U_{i}(\phi)=\gamma_{i}^{-1} I_{i}\left(1-\exp \left(-\gamma_{i} \phi\right)\right)$ [15].

It is convenient to investigate the dynamics in a return map by choosing an arbitrary oscillator as reference 22]. Here the oscillator 1 is chosen. When the reference oscillator is reset, the phases are recorded. The number of reset times is used as time step for the return map. The dynamics can be simulated by an event-by-event based numerical calculation which can be applied to obtain the solutions for the pulse-coupled network with delay [20].

We choose the similar parameter values as in Ref. [15], where the existence of stable irregular transients is analytically proved, i.e., $\gamma_{i}=1, I_{i}=4.0, b=1, \epsilon=-1.6$,
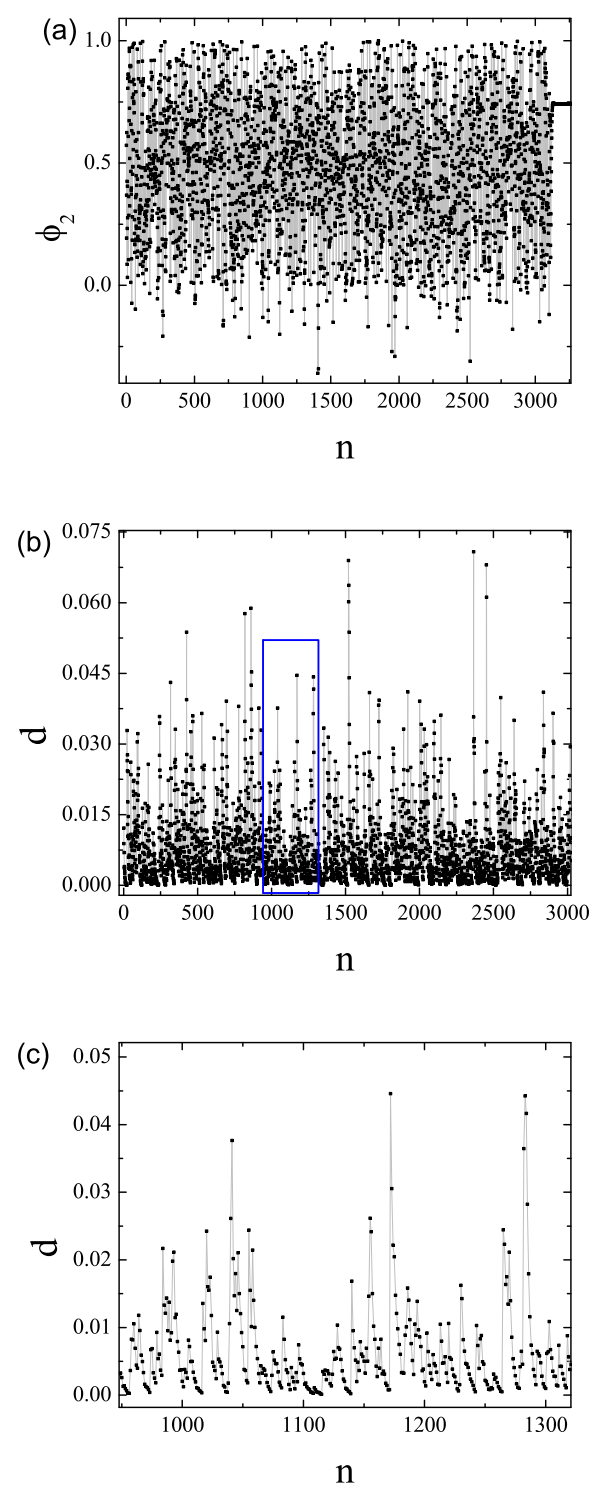

FIG. 2: (a) The long irregular transients observed for pulsecoupled oscillators for $\phi_{2}$ using oscillator 1 as reference. The $n$ represents the number of times the reference oscillator has been reset. (b) The corresponding distance sequence $d$ to the basin boundaries. (c) The enlargement of the rectangle in (b).

and $\tau=0.1$. The network size is fixed at $N=20$. We observed that long irregular transients exist when diluting links from fully coupled networks. Fig. 2(a) shows one typical transient trajectory for connection probability $p=0.2$. From the distance sequence to the basin boundaries, as shown in Figs. 2(b) and 2(c), once again, we find the similar pattern as shown in Fig. 1. During transients, the trajectory repeatedly goes to the basin boundaries and then jumps to some remote regions in the phase space. The existence of the same regular pattern in the above two different systems strongly suggests that the formation of stable chaos in different discon- 
tinuous dynamical systems actually could have the same dynamical origin. We emphasize that such pattern is unique in stable chaos, and does not exist in chaotic transients observed in the excitory pulse-coupled oscillators [21], which should have a different mechanism.

\section{DYNAMICAL FORMATION OF STABLE CHAOS}

In Sec. II, we observe a regular pattern in the stable chaos by measuring the distance to the basin boundaries for each point in the transient trajectory. This regular pattern displays the internal structure of the stable irregular transients. During the transient period, the trajectory repeatedly goes to different regions on the basin boundaries. This seems to suggest that there is a path that can connect two different places on the basin boundaries. Through extensive numerical experiments, we confirm the existence of such paths. Furthermore, it is found that the ending point of such a path is an image of the starting point, and both the starting point and the ending point are on the discontinuous boundaries. We thus call this path a guiding path. The dynamical formation of this path is through the intersection of discontinuous boundaries with their images. It can be easily verified that the transient structure in the system (10) is formed in this way where the discontinuous boundaries are $x_{i}=0.98$. The dynamical origin of stable chaos lies in the formation of many guiding pathes in phase space. At the starting and the ending points, the dynamics are discontinuous. Between these two points, the trajectory usually follows the contracting dynamics which results in the decreasing distance to the basin boundaries.

To verify the above mechanism of the formation of stable chaos, we need to locate guiding paths and then compare with the corresponding jumping processes from one region to another region on the basin boundaries. For high dimensional systems, such as Eq. (1), we can obtain approximately the underlying guiding path by sampling many initial points, say $10^{6}$, in a small region where a jumping process starts. The length of guiding path $n$ usually is the same as the jumping process. We can obtain all these trajectories with length $n$. Then we approximate the guiding path by a trajectory with minimum quantity $D$, where $D$ is defined to be the sum of distance of two endpoints of a trajectory to the discontinuous boundaries. In this way, we can verify that there is a guiding path governing the jumping process from one region of basin boundaries to another region.

To be more accurate and more reliable, here we construct a two-dimensional map and develop a numerical technique to obtain the guiding paths with high accuracy. The dynamical equations of the discontinuous maps are:

$$
\begin{aligned}
& x_{1}=e f\left(x_{1}\right)+(1-e) f\left(x_{2}\right), \\
& x_{2}=e f\left(x_{2}\right)+(1-e) f\left(x_{1}\right),
\end{aligned}
$$

where the local map $f$ is shown in Fig. 3, which can be regarded as the generalized map used in Eq. (1) with more discontinuous boundaries.

In the following, we specifically describe the technique to locate the guiding paths accurately for the twodimensional maps $F$ whose discontinuous boundaries are composed of straight lines. The key procedure of this technique is to locate the guiding paths with given length $n$ and an initial interval $[A, B]$. By varying $n$ and the interval, we can locate all the guiding paths of interest. To this end, we divide the phase space into squares, or cells by the discontinuous boundaries. An interval $[A, B]$ may contain starting points of the guiding paths if $F^{n}(A)$ and $F^{n}(B)$ fall into two different cells. Consider the midpoint $m=(A+B) / 2$, we can find a smaller interval $[A, m]$ or $[m, B]$ whose $n$th images of the two endpoints fall into two different cells. We can recursively apply this bisection method to shrink the interval and obtain an interval $[a, b]$ with given accuracy, say, $10^{-6}$. Then one $n$th image of the two endpoints will be the ending point of the guiding path if it is within the distance $10^{-6}$ of discontinuous boundaries. After that, we consider the remaining interval $[b, B]$. In this way, we can find all guiding paths with length $n$ with starting points belonging to $[A, B]$.

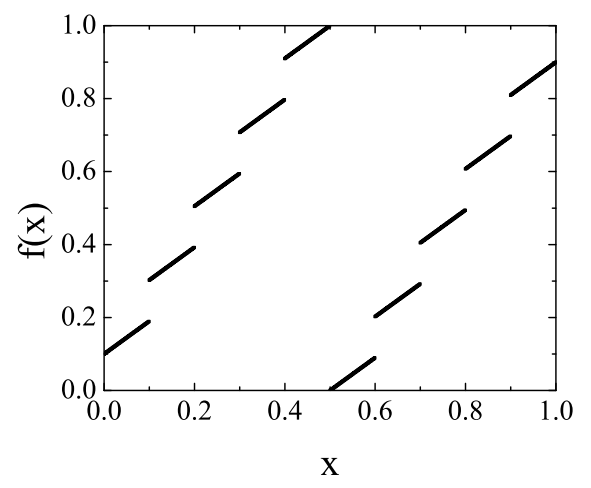

FIG. 3: A map with only contracting pieces and many discontinuous boundaries.

A typical transient trajectory in Eq. (4) is shown in Fig. 4(a) for $e=0.09$. In the distance sequence, as shown in Fig. 4(b), a regular pattern similar to those in Figs. 1(c) and 2(c) is much more evident. Here, since our system is only two-dimensional, it is easy for us to directly verify our analysis of the mechanism that led to the formation of stable chaos. In Fig. 5, we plot part of the transient trajectory within a specific time window, i.e., from $n=18$ to $n=35$. As shown in Fig. 4(c), during this time period, the transient trajectory goes from one region of the basin boundary to another region of the basin boundary. In Fig. 5, we plot both the transient trajectory from $n=18$ to $n=35$, and a guiding path. Remarkably, it is clearly seen that the transient trajectory exactly follows the guiding path going from one point of the basin boundary to another point on the basin boundary. Since there exist plenty of these guiding pathes in the phase space, we can expect long irregular 
transients to occur in such coupled map systems, especially when the dimension of the coupled system is very high.
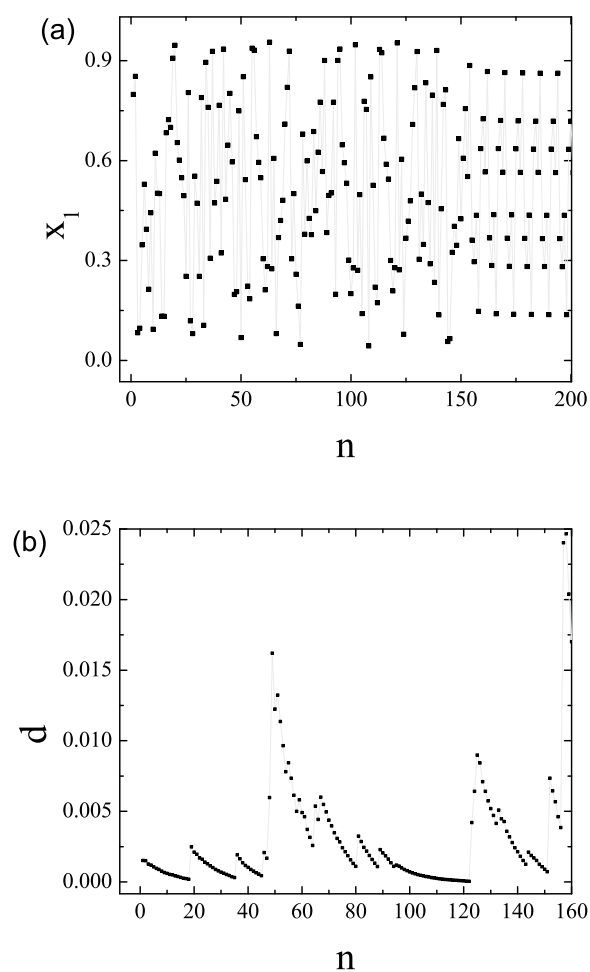

FIG. 4: A typical transient trajectory (a) for Eq. (2) and its corresponding distance sequence (b).

It is interesting to compare stable chaos to the transient chaos usually occurring in continuous dynamical systems. The skeleton for transient chaos is the infinite number of unstable periodic orbits (UPOs) embedded in the chaotic saddle. While for the stable irregular transient or stable chaos, the underlying microscopic structure is the guiding path with both starting and ending points on the discontinuous boundaries. A guiding path is not a cyclic structure, i.e. the starting and ending points are not the same, which is the major difference with an UPO. The regular patterns occurring in the high dimensional systems Eq. (11) and Eq. (2) impliy that the guiding paths are clustered in phase space, which is similar to the dense UPOs. Here, the clustered guiding paths are generally associated with the large number of discontinuous boundaries in the high dimensional systems. For Eq. (1), the number of discontinuous boundaries is $N+\left(\begin{array}{c}N \\ 2\end{array}\right)+\left(\begin{array}{c}N \\ 3\end{array}\right)+\cdots$. It is expected that this fast growing of the number of discontinuous boundaries with dimension will make the guiding paths more clustered in high dimensional systems, somewhat similar to the attractor crowding effect 24]. In turn, it will make stable chaos more easily observed in high dimensional systems.
From the microscopic structure, we can also under-

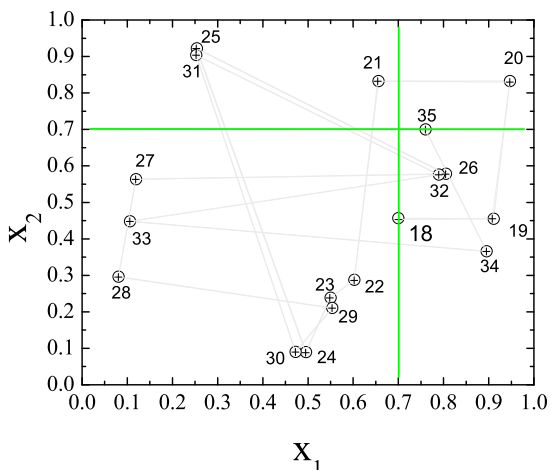

FIG. 5: Part of transient trajectory shown in plus and a guiding path shown in circle from $n=18$ to $n=35$. The two lines represent two discontinuous boundaries. The number represents the the time step $n$.

stand why stable chaos is unstable against finite small perturbation (stable against infinitesimally small perturbation) [16]. During the long transient time, stable chaos takes places near many guiding paths whose starting and ending points belong to the basin boundaries.

\section{CONCLUSION}

In this paper, we investigate the dynamical formation of long irregular transients with negative largest Lyapunov exponent, i.e., the stable chaos, directly based on the dynamical equations. We show that these irregular transients actually have certain structure which can be illustrated by the distance sequence to the basin boundaries. The transients repeatedly approaches the basin boundaries and then jumps from the boundaries to a remote region in the phase space. Through numerical simulations, it is shown that there exists a guiding path whose ending point is an image of the starting point and both of them are on the discontinuous boundaries. It is these guiding pathes that connect different points on the basin boundaries, making the dynamics of the system exhibit long irregular behavior before it goes to the final stable attractor. Thus the present work reveals a mechanism for the formation of stable chaos in coupled discontinuous map systems.

\section{Acknowledgments}

This work is supported by the National University of Singapore, and DSTA of Singapore under Project Agreement POD0613356. 
[1] T. Tel and Y. C. Lai, PHYS. REP. 460, 245 (2008).

[2] C. Grebogi, E. Ott and J. A. Yorke, Physica D 7, 181 (1983).

[3] C. Grebogi, E. Ott and J. A. Yorke, Phys. Rev. Lett. 50, 935 (1983).

[4] C. Grebogi, E. Ott and J. A. Yorke, ERGOD. THEOR. DYN. SYST. 5, 341 (1985).

[5] Y. C. Lai and R. L. Winslow, Phys. Rev. Lett. 74, 5208 (1995).

[6] Y. Do and Y. C. Lai, Europhys. Lett. 67, 914 (2004).

[7] Y. Do and Y. C. Lai, Phys. Rev. E 71,046208 (2005).

[8] J. P. Crutchfield and K. Kaneko, Phys. Rev. Lett. 60, 2715 (1988).

[9] A. Politi, R. Livi, G.-L. Oppo and R. Kapral, Europhys. Lett. 22, 571 (1993).

[10] A. Politi and A. Torcini, Europhys. Lett. 28, 545 (1994).

[11] Y. Cuche, R. Livi and A. Politi, Physica D 103, 369 (1997).

[12] F. Ginelli, R. Livi and A. Politi, J. Phys. A: Math. Gen. 35, 499 (2002).

[13] R. Bonaccini and A. Politi, Physica D 103, 362 (1997).

[14] R. Zillmer, R. Livi, A. Politi and A. Torcini, Phys. Rev.
E 74, 036203 (2006).

[15] S. Jahnke, R. M. Memmesheimer and M. Timme, Phys. Rev.Lett. 100, 048102 (2008).

[16] R. Zillmer, N. Brunel and D. Hansel, Phys. Rev. E 79, 031909 (2009).

[17] F. Bagnoli and R. Rechtman, Phys. Rev. E 73, 026202 (2006).

[18] S. V. Ershov and A. B. Potapov, Physics Letters A 167, 60 (1992).

[19] R. E. Mirollo and S. H. Strogatz, SIAM J. Appl. Math. 50, 1645 (1990).

[20] M. Timme, F. Wolf and T. Geisel, Chaos 13, 377 (2003).

[21] A. Zumdieck, M. Timme, T. Geisel and F. Wolf, Phys. Rev.Lett. 93, 244103 (2004).

[22] M. Timme, F. Wolf and T. Geisel, Phys. Rev. Lett. 89,154105 (2002).

[23] U. Ernst, K. Pawelzik and T. Geisel, Phys. Rev. Lett. 74, 1570 (1995).

[24] K. Wiesenfeld and P. Hadley, Phys. Rev. Lett. 62, 1335 (1989). 